\title{
Quale terapia per la prevenzione della nefrolitiasi
}

\author{
Commento all'articolo: "Medical management to prevent recurrent nephrolithiasis in adults: \\ a systematic review for an American College of Physicians Clinical Guideline"
}

\section{Maurizio Terribile, Roberta Rossano, Mario Paracuollo, Marco Terribile}

\author{
U.O.C. di Nefrologia e Dialisi, Ospedale dei Pellegrini, Napoli
}

La Nefrolitiasi (NL) è una patologia la cui prevalenza è raddoppiata negli ultimi 30 anni (1). Il rischio di un episodio di NL nella popolazione generale è di circa il $10 \%$ (2). La probabilità di recidiva è intorno al $50 \%$ a 10 anni (3). È pertanto di notevole importanza una adeguata terapia per la prevenzione delle recidive.

I dati sulla efficacia sia delle misure dietetiche che della terapia medica nella prevenzione della NL non sono però univoci.

È stata recentemente pubblicata (4) una interessante metanalisi sulla terapia per la prevenzione della nefrolitiasi.

Sono stati valutati 28 studi randomizzati e controllati pubblicati entro settembre 2012.

Nell'ambito di questi studi, 8 valutavano l'efficacia dell'intervento dietetico mentre 20 quella dell'intervento farmacologico. Gli Autori hanno giudicato 2 studi come di alta qualità, 2 come di bassa qualità e gli altri 24 come di qualità media.

In riferimento all'intervento dietetico è stato riscontrato, con la terapia idropinica, un ridotto rischio di recidive di NL solo in 1 studio di "bassa qualità" mentre nell'altro studio "di media qualità" i risultati non sono stati statisticamente significativi. Gli studi riguardanti l'apporto di "soft drinks", di una dieta ricca di fibre o ipoproteica, o di una dieta "multicomponente" hanno dato risultati discordanti e comunque non conclusivi.

Sei studi "di media qualità" hanno complessivamente registrato una maggiore efficacia del diuretico tiazidico rispetto al placebo nel ridurre le recidive di NL.

Cinque studi di "media qualità" e 1 di "alta qualità" hanno riportato un'azione positiva dei citrati nella riduzione del rischio di recidive. Quattro studi “di media qualità" hanno complessivamente registrato la maggiore efficacia dell'allopurinolo rispetto al placebo nel ridurre le recidive di NL.

In tutti questi studi di intervento farmacologico molti pazienti non hanno però portato a termine lo studio. Inoltre, i dati biochimici urinari erano spesso riportati solo parzialmente.

Dopo questa importante metanalisi vi sono stati pochi e limitati trials.

È stato pubblicato nel novembre 2012 da Herrel et al (5) un interessante studio su una bevanda di uso comune quale la Coca Cola. Sono stati studiati i fattori urinari di rischio litogeno. Lo studio metabolico non ha evidenziato alcun aumento dei fattori di rischio rispetto a chi assumeva solo acqua.

Wolfgram et al (6) hanno pubblicato nel 2013 uno studio che ha valutato comparativamente l'efficacia di 2 diuretici tiazidici quali l'idroclorotiazide e il clortalidone nella riduzione della ipercalciuria. I loro dati hanno evidenziato una maggiore efficacia del clortalidone.

È stato successivamente pubblicato nel novembre 2013 da Goldfarb et al (7) uno studio di 6 mesi che ha valutato comparativamente l'efficacia del febuxostat (Adenuric) e del-l'allopurinolo nel ridurre l'iperuricuria e il rischio di recidive di NL. Il febuxostat ha ridotto significativamente l'escrezione urinaria di acido urico ma non il rischio di recidive. Il periodo di studio era però molto breve. È stato infine pubblicato nel marzo 2014 uno studio di Noori et al (8), dove sono stati studiati 57 pazienti con iperossaluria e NL valutando comparativamente l'efficacia di una dieta a basso contenuto di ossalati rispetto alla dieta DASH (Dietary Approaches to stop Hypertension).

La dieta DASH consiste in una dieta ricca di frutta, vegetali e povera di grassi, carni e dolci. La dieta DASH ha comportato una più elevata escrezione urinaria di ossalato ma una più bassa saturazione per il $\mathrm{CaOx}$; quest'ultimo dato è correlato ad una aumentata escrezione urinaria di citrato, magnesio e ad un aumento del $\mathrm{pH}$.

Pertanto la dieta DASH si è rivelata ugualmente efficace rispetto a quella a basso contenuto di ossalati.

In conclusione, non vi sono a tutt'oggi dati certi e concordanti a favore di uno specifico intervento dietetico e/o farmacologico per la prevenzione delle recidive da NL.

Ė comunque difficile approntare studi rigorosi, con un sufficiente numero di soggetti reclutati, per un numero sufficiente di anni e senza bias che molto spesso inficiano la qualità dei risultati. Pertanto molto spesso si prendono in considerazione "endpoint surrogati" quali le variazioni di singoli parametri biochimici rispetto all'“endpoint clinico maggiore" rappresentato dalle recidive di NL.

È in ogni caso consigliabile mettere in atto la terapia idropinica e quella farmacologica con l'utilizzo di citrati, tiazidici, allopurinolo. 
Dichiarazione di conflitto di interesse: Gli Autori dichiarano di non avere conflitto di interessi.

Contributi economici agli Autori: Gli Autori dichiarano di non avere ricevuto sponsorizzazioni economiche per la preparazione dell'articolo.

Indirizzo degli Autori:

Dr. Maurizio Terribile

U.O.C. di Nefrologia e Dialisi

Ospedale dei Pellegrini

Via Portamedina alla Pignasecca 41

80134 Napoli

maurizio.terribile@gmail.com

\section{Bibliografia}

1. Romero V, Akpinar H, Assimos DG. Kidney stones: a global picture of prevalence, incidence, and associated risk factors. Rev Urol 2010; 12: e86-e96.

2. Lieske JC, Peña de la Vega LS, Slezak JM, et al. Renal stone epidemiology in Rochester, Minnesota: an update. Kidney Int 2006; 69: $760-4$.

3. Uribarri J, Oh MS, Carroll HJ. The first kidney stone. Ann Intern Med 1989; 111: 1006-09.

4. Fink HA, Wilt TJ, Eidman KE, Garimella PS. Medical Management to prevent Nephrolithiasis in Adults: A systematic Review for an American College of Physicians Clinical Guidelines. Ann Intern Med 2013; 158: 535-43.

5. Herrel L, Pattaras J, Solomon T, Ogan K. Urinary stone risk and cola consumption. Urology 2012; 80(5): 990-4.

6. Wolfgram DF, Gundu V, Astor BC, Jhagroo RA. Hydrochlorothiazide compared to chlorthalidone in reduction of urinary calcium in patients with kidney stones. Urolithiasis 2013; 41(4): 315-22.

7. Goldfarb DS, MacDonald PA, Gunawardhana L, Chefo S. Randomized controlled trial of febuxostat versus allopurinol or placebo in individuals with higher urinary uric acid excretion and calcium stones. Clin J Am Soc Nephrol 2013; 8(11): 1960-7.

8. Noori N, Honarkar E, Goldfarb DS, et al. Urinary lithogenic risk profile in recurrent stone formers with hyperoxaluria: a randomized controlled trial comparing DASH (Dietary Approaches to Stop Hypertension)-style and low-oxalate diets. Am J Kidney Dis 2014; 63(3): 456-63. 